\title{
Efecto de la suplementación con Sambucus nigra, oreado y ensilado, sobre parámetros productivos y económicos de conejos de engorde
}

\section{Effect of Supplementation with wilted and silaged Sambucus nigra on productive and economic parameters of rabbits in fattening phase}

\author{
Jenny Marcela Moreno Poveda ${ }^{1}$; Fallon Yamile Riaño Jiménez ${ }^{2}$
}

\begin{abstract}
'Zootecnista, M.Sc. Universidad de Ciencias Aplicadas y Ambientales, Facultad de Ciencias Agropecuarias. Bogotá, D.C., Colombia; e-mail: marcelamoreno@ udca.edu.co; (D) https://orcid.org/0000-0002-4390-673X

2Zootecnista, M.Sc. Universidad de Ciencias Aplicadas y Ambientales U.D.C.A, Facultad de Ciencias Agropecuarias. Bogotá, D.C., Colombia; e-mail: friaño@ udca.edu.co; (D) https://orcid.org/0000-0001-6797-5193
\end{abstract}

Cómo citar: Moreno Poveda, J.M.; Riaño Jiménez, F.Y. 2020. Efecto de la suplementación con Sambucus nigra, oreado y ensilado, sobre parámetros productivos y económicos de conejos de engorde. Rev. U.D.C.A Act. \& Div. Cient. 23(1):e1468. http://doi.org/10.31910/ rudca.v23.n1.2020.1468

Artículo de acceso abierto publicado por Revista U.D.C.A Actualidad \& Divulgación Científica, bajo una licencia Creative Commons CC BY-NC 4.0

Publicación oficial de la Universidad de Ciencias Aplicadas y Ambientales U.D.C.A, Institución de Educación Superior Acreditada de Alta Calidad por el Ministerio de Educación Nacional.

Recibido: Febrero 8 de 2020

Aceptado Mayo 10 de 2020

Editado por: Ingeborg Zenner de Polanía

\section{RESUMEN}

Con el objetivo de disminuir los costos de alimentación en cunicultura, se evaluó la inclusión de sauco oreado y ensilado sobre el desempeño productivo y económico de conejos en fase de engorde. Fueron seleccionados 27 conejos (cruce entre Chinchilla, Californiano y Nueva Zelanda), con peso inicial promedio de 1467,6 $\pm 271,1 \mathrm{~g}$. Éstos, se dividieron y se asignaron al azar en cada uno de los tres tratamientos, control $(100 \%$ concentrado), ensilaje $(85 \%$ concentrado $+15 \%$ ensilaje de sauco) y oreado $(88 \%$ concentrado + $12 \%$ sauco oreado). Durante 28 días, se evaluó consumo, ganancia de peso, factor de conversión alimenticia (FCA), índice viscerosomático y rendimiento en canal, para, lo cual, se realizó un análisis de varianza en un diseño completo al azar. Se calculó el margen bruto de ingreso parcial (MBIP), a través de un análisis de presupuesto parcial para la venta del producto, en pie y en canal. El grupo que recibió el sauco ensilado expresó una mayor ganancia de peso $(1040,4 \pm 75,2 \mathrm{~g})$ y un mayor consumo $(12,5 \pm 0,3 \mathrm{~kg} \mathrm{MS} /$ jaula $)$, respecto al grupo control $(\mathrm{P}<0,05)$; en contraste, en el FAC, no se observó diferencia entre estos dos grupos $(\mathrm{P}<0,05)$. En los parámetros productivos restantes no se observaron diferencias estadísticas $(\mathrm{P}>0,05)$. La elaboración del ensilaje presentó el mayor costo por concepto de alimentación, pero, a la vez, generó el mayor MBIP, para la venta en pie $(13 \%)$ y en canal (9\%). El ensilaje de sauco puede ser considerado como una alternativa de alimentación, mejorando los parámetros productivos y el MBIP.

Palabras clave: ingredientes alternativos; saúco; cunicultura; plantas de ramoneo; costos de producción. 


\section{ABSTRACT}

In order to reduce the feeding costs in cuniculture, the inclusion of wilted and silaged elder was evaluated on the productive and economic performance of rabbits in the fatting phase. 27 rabbits (crosses between Chinchilla, Californian and New Zealand) were selected with an average initial weight of $1467,6 \pm 271,1 \mathrm{~g}$. They were divided and randomly distributed in each of three treatments, control (100\% balanced feed), silage ( $85 \%$ balanced feed $+15 \%$ elder silage) and wilted ( $88 \%$ balanced feed $+12 \%$ wilted elder). During 28 days, weight gain, feed conversion ratio (FCR), carcass yield and viscerosomatic index were evaluated and analyzed through a variance analysis in a complete random design. Gross margin of partial income (GMPI) was calculated through a partial budget analysis for the sale of the product in vivo and carcass. The group that received the silage showed greater weight gain $(1040.4 \pm 75.2 \mathrm{~g})$ and higher intake $(12.5 \pm 0.3 \mathrm{~kg}$ MS / cage), than control group $(\mathrm{P}<0,05)$, but FCR was not different between these groups $(\mathrm{P}<0.05)$. No statistical differences were observed in the remaining productive parameters $(\mathrm{P}>0,05)$. Silage diet presented the highest cost, but at the same time it produced the highest GMPI, for sale in vivo $(13 \%)$ and carcass $(9 \%)$. Silage elder can be considered as a feeding alternative, improving the productive parameters and the GMPI.

Keywords: alternative ingredients; elder; rabbit production; browse plants; production costs.

\section{INTRODUCCIÓN}

La cunicultura es considerada como una actividad favorable para campesinos en todo el mundo, debido a su fácil manejo, la rapidez para recuperar la inversión y la posibilidad de generar ingresos modestos durante el año (Olivares et al. 2009). Al respecto, Lebas et al. (1996) resaltan diversas ventajas, como individuos ideales para un sistema de producción, ya que, al ser herbívoros, no compiten por los recursos disponibles para la alimentación humana, son altamente prolíficos y su sistema de producción requiere bajo costo de inversión y mano de obra.

Adicionalmente, se han reportado las ventajas en el consumo, por las características nutricionales de su carne, la cual, es baja en grasa y colesterol, presentando altos niveles de proteína (Cullere \& Dalle, 2018; Lebas, 1988); asimismo, con enriquecimiento dietario, se puede mejorar aún más su perfil nutricional (Dalle \& Szendrő, 2011).

Las características antes mencionadas son relevantes en el contexto colombiano, ya que son de interés para la búsqueda de la seguridad y la soberanía alimentaria. Bajo las circunstancias actuales, en el que el aumento de la población y el bajo ingreso económico de los sectores menos favorecidos aumentan la vulnerabilidad ante la baja disponibilidad de alimentos y su elevado precio (Barrial \& Barrial, 2011), la producción de especies menores, como la cunicultura, se consideran una solución apropiada para abastecer el consumo de proteína de origen animal, por parte de la poblaciones campesinas y vulnerables (Seija, 2011).
Teniendo en cuenta lo anterior y las características fisiológicas y hábitos alimenticios del conejo, se justifica la inclusión de suplementos vegetales, bajo condiciones de producción en confinamiento, que permitan disminuir el uso de concentrados comerciales, generalmente, de mayor costo, que pueden poner en riesgo la rentabilidad del proceso productivo.

En este sentido, el sauco (Sambucus nigra) se muestra como una alternativa de fácil acceso para la suplementación, siendo una planta arbustiva, que ha resultado promisoria, como banco forrajero, por su amplia distribución a nivel mundial, puesto que a pesar de ser nativo de Europa, noroeste de África y sudoeste de Asia, en Colombia, se encuentra distribuido en los departamentos de Boyacá, Caldas, Putumayo, Quindío, Antioquia, Cauca, Cundinamarca, Valle del Cauca, Nariño, Amazonas y Huila, en altitudes que varían entre 1.400 y 2.600 m s.n.m. (MAVDT, 2009).

En cuanto a su composición nutricional, Carvajal \& Cuesta (2016) reportan porcentajes de proteína total entre 15,2 y 25,8 y contenido de pared celular que varía entre 19 y 38,9\%, perfilándolo como un recurso forrajero de alto valor nutricional y con potencial para ser incluido en la dieta de especies herbívoras (Cárdenas et al. 2011), como material fresco o conservado (Sánchez et al. 2009). El proceso de ensilaje, ha sido catalogado como el método más apropiado de conservación para el sauco, debido a que se preserva su calidad nutricional (Carvajal \& Cuesta 2016).

El presente estudio evaluó el efecto de la inclusión de sauco fresco y ensilado sobre el desempeño productivo y económico de conejos en fase de engorde.

\section{MATERIALES Y MÉTODOS}

Los procedimientos y el diseño experimental aquí expuestos fueron aprobados por el comité de ética de la facultad de Ciencias Agropecuarias de la Universidad de Ciencias Aplicadas y Ambientales U.D.C.A; asimismo, se respetaron las normas de bienestar animal, en el transcurso del ensayo.

Ubicación. El estudio, se llevó a cabo en las instalaciones de la Unidad Experimental El Remanso, de la U.D.C.A, ubicada en Bogotá, Colombia, a $2.560 \mathrm{~m}$ s.n.m., $4^{\circ} 35^{\prime}$ latitud Norte y $74^{\circ}$ 04) longitud Oeste y temperatura promedio de $14^{\circ} \mathrm{C}$.

Manejo de animales. Se utilizaron 27 conejos, producto de cruces entre las razas Chinchilla, Californiano y Nueva Zelanda, en fase de levante (3-4 semanas pos destete), con peso inicial promedio de 1467,6 $\pm 271,1 \mathrm{~g}$. Fueron adquiridos del sistema de producción cunícola del SENA sede Mosquera. Previo al periodo experimental, los animales fueron distribuidos aleatoriamente y de manera homogénea en 9 jaulas. A partir de su llegada, durante el periodo de adaptación (15 días) y en la etapa experimental (28 días), se les suministró agua y alimento a voluntad. Los animales fueron pesados semanalmente en horas de la mañana, antes del suministro del alimento. 
Previo al sacrificio, los animales permanecieron en ayuno por 24 horas, con el fin de realizar el vaciamiento gástrico. El sacrificio fue realizado mediante insensibilización y degüelle.

Dietas experimentales. Se utilizó hojas y peciolos de sauco con $22 \%$ de materia seca, 5,6\% de proteína y $1000 \mathrm{Kcal}$ de energía bruta $(\mathrm{EB}) / \mathrm{kg}$, en base húmeda, en dos tipos de presentación, oreado y ensilado. El sauco oreado fue expuesto previamente al aire libre durante 48 - 72 horas, bajo sombra. La elaboración del ensilaje incluyó 2,5\% de melaza, 2,5\% de glice rol y 95\% de saúco, en base húmeda. El proceso de fermentación anaeróbica duró, aproximadamente, 28 días, tiempo, en el cual, se midió pH y amonio, como parámetros de calidad. Junto con el sauco (oreado o ensilado), se ofreció concentrado comercial, con $87 \%$ de materia seca, $14,8 \%$ de proteína cruda y $2867 \mathrm{Kcal}$ de EB/kg de dieta, en base húmeda.

A continuación, se describen las dietas experimentales:

Dieta 1 (Control). 100\% concentrado comercial

Dieta 2 (Ensilaje). 85\% concentrado comercial $+15 \%$ ensilaje de sauco.

Dieta 3 (Oreado). 88\% concentrado comercial $+12 \%$ sauco oreado.

Los tres materiales fueron ofrecidos a voluntad. Los porcentajes de inclusión mostrados fueron calculados al finalizar el periodo experimental y se presentan como porcentaje de materia seca consumida.

Parámetros evaluados. Los parámetros productivos evaluados fueron peso final, ganancia de peso (peso final - peso inicial), consumo ( $\mathrm{kg}$ MS/jaula) y factor de conversión alimenticia (consumo de alimento/ganancia de peso). En los parámetros económicos, con el fin de encontrar el margen bruto de ingreso parcial (MBIP) de cada uno de los tratamientos, se realizó un análisis de presupuesto parcial, teniendo en cuenta el costo de alimentación durante la fase experimental y la cantidad de carne producidos (kg); para lo cual, se asumieron dos escenarios, el primero, con una comercialización de animales en pie y, la segunda, en canal. Asimismo, se calculó el costo por kilogramo de materia seca para el concentrado comercial, sauco fresco y ensilado, que fueron multiplicados por la cantidad de alimento consumido en materia seca por los 9 conejos que componían las 3 unidades experimentales de cada tratamiento y así se obtuvo el costo total por concepto de alimentación, para el periodo evaluado. Para el cálculo de la cantidad de carne disponible para la venta en pie, se calculó el total de la biomasa ganada por tratamiento durante los días evaluados, con el fin de obtener el costo por kilogramo de carne producida, por lo que se usó la siguiente fórmula: costo total de alimentación/biomasa ganada. Para el caso de la venta en canal, se tomó la biomasa final y se multiplicó por el rendimiento en canal, con el fin de obtener la cantidad disponible; posteriormente, se estimó un precio de venta de $\$ 10.000 / \mathrm{kg}$, cuando el animal es comercializado en pie y $\$ 16.000 / \mathrm{kg}$, cuando se comercializa en canal, con lo cual, fue posible calcular el ingreso total y después obtener el MBIP de cada escenario. Este parámetro, se obtuvo descontando del ingreso total el costo de alimentación parcial obtenido. El paso seguido, se estimó el margen de utilidad sobre el ingreso (MUI), que indica el porcentaje de ganancia parcial, obtenido del ingreso total al descontar el costo total de alimentación. Por último, se calculó el valor diferencial en pesos, que refleja en cuánto aumenta o disminuye la ganancia obtenida (\$), utilizando como punto de referencia el MBIP, obtenido con la dieta control.

Diseño experimental y análisis estadístico: El experimento fue realizado bajo un diseño completamente al azar, evaluando 3 tratamientos (dietas experimentales), cada uno con tres repeticiones (jaula), que contenían 3 conejos, para un total de 27 animales.

Previa verificación de los supuestos de homogeneidad de las varianzas (prueba de Levene) y normalidad del error (prueba de Shapiro-Wilk) (Martínez et al. 2011), los datos fueron sometidos a análisis de varianza ANOVA, donde el nivel de significancia fue del $5 \%$. Cuando se evidenciaron diferencias significativas $(\mathrm{P}<0,05)$, las medias fueron comparadas mediante la prueba de Tukey. Para el análisis de los datos, se utilizó el programa estadístico SAS V. 9.0. (SAS Institute, 2002).

\section{RESULTADOS Y DISCUSIÓN}

Parámetros productivos: Dentro de los resultados obtenidos, se destaca el grupo que recibió sauco ensilado, con una mayor ganancia de peso, al compararlo con los demás tratamientos $(\mathrm{P}<0,05)$, (Tabla 1).

$\mathrm{Al}$ respecto, en la literatura consultada, se evidencia una limitante en la información reportada acerca de estudios que incluyan la respuesta de conejos en fase de engorde, bajo suplementación con diferentes fuentes forrajeras, propias de los ecosistemas tropicales, sus combinaciones y su impacto económico (Mbanya et al. 2005).

En el caso del sauco, la literatura muestra algunas características nutricionales, como el nivel de fibra en detergente neutro (FDN) $(37,4 \% \pm 0,91)$, además de un alto potencial para la alimentación animal en general (Carvajal et al. 2012; Nova et al. 2005); por ejemplo, en ganadería de leche, esta arbórea puede reemplazar el $20 \%$ del concentrado, obteniendo producciones de leche de $94 \%$ respecto a la obtenida con dietas $100 \%$ concentrado, con un ahorro de 0,24 USD/vaca/día (Carvajal et al. 2012); sin embargo, la información nutricional en la alimentación de conejos con este material es escasa, pero se reporta que la sustitución del almidón en la dieta por fibra digestible (hemicelulosa) hasta un 50\% no afecta el consumo ni la ganancia de peso de los conejos, sino que, por el contrario, es posible incrementar un 60\% de la degradabilidad de la FDN, efectuando este tipo de sustitución (Gidenne \& Jehl, 1996).

García et al. (2006) reportan que la inclusión de fuentes de fibra más solubles en las dietas de los conejos aumenta la longitud de las vellosidades intestinales y, por el contrario, la inclusión de fuentes de fibra lignificada pueden producir atrofia en la estructura del tejido y alterar el funcionamiento normal de las células epiteliales del intestino, encargadas de romper diversas moléculas alimenticias y transportarlas al interior de los enterocitos intestinales. 
Tabla 1. Parámetros productivos de conejos en etapa de engorde alimentados con sauco fresco o ensilado, durante 28 días.

\begin{tabular}{|l|l|l|l|}
\hline \multicolumn{1}{|c|}{ Parámetro } & \multicolumn{1}{c|}{ Control } & \multicolumn{1}{c|}{ Oreado } & \multicolumn{1}{c|}{ Ensilaje } \\
\hline Peso final (kg)/conejo & $2303,6 \pm 406,5^{\mathrm{A}}$ & $2359,4 \pm 178,3^{\mathrm{A}}$ & $2545,0 \pm 284,9^{\mathrm{B}}$ \\
\hline Ganancia de peso/ animal (kg) & $824,3 \pm 63,5^{\mathrm{A}}$ & $853,7 \pm 83,7^{\mathrm{A}}$ & $1040,4 \pm 75,2^{\mathrm{B}}$ \\
\hline Consumo (kg MS/jaula) & $10,7 \pm 0,7^{\mathrm{A}}$ & $11,7 \pm 0,6^{\mathrm{AB}}$ & $12,5 \pm 0,3^{\mathrm{B}}$ \\
\hline Conversión alimenticia & $3,9 \pm 0,4^{\mathrm{A}}$ & $4,7 \pm 0,3^{\mathrm{B}}$ & $4,0 \pm 0,1^{\mathrm{AB}}$ \\
\hline Rendimiento en Canal & $48,5 \pm 1,7$ & $49,8 \pm 3,0$ & $49,0 \pm 3,0$ \\
\hline Índice Viscerosomático & $19,7 \pm 1,1$ & $18,8 \pm 1,6$ & $21,8 \pm 1,7$ \\
\hline
\end{tabular}

Letras diferentes indican diferencias significativas mediante el Test de Tukey, a un nivel de significancia del 5\%.

Por su parte, Lebas (1988) menciona que la relación de transformación promedio de proteína vegetal a proteína animal en conejos oscila entre el 18 y el $22 \%$, cuando son alimentados con materiales fibrosos, cuya variación depende del potencial productivo del animal. En este sentido, se debe considerar la importancia de la proteína y la fibra en el tracto digestivo del conejo, ya que estos participan en procesos importantes, como la recirculación de los nutrientes (cecotrofía), hasta obtener, del alimento inicial, el máximo provecho; esta característica fisiológica del conejo le permite la conversión de alimentos con relativo bajo valor para el ser humano, en proteína muscular de alto valor nutricional (Mora-Valverde, 2010).

En la variable consumo del presente estudio, se observaron diferencias significativas entre tratamientos $(\mathrm{P}<0,05)$, donde los animales alimentados con sauco ensilado presentaron un mayor valor al compararlo con el tratamiento control, cuya diferencia fue del 17\%; sin embargo, al analizar el parámetro de conversión alimenticia no se observaron diferencias entre estos dos tratamientos, debido a la mayor ganancia de peso encontrada en los animales que recibieron el sauco ensilado, indicando una mayor eficiencia productiva.

Lo anterior tiene relación con lo reportado por Garcés et al. (2004), quienes afirman que la fermentación láctica que realizan los microorganismos, durante el proceso de ensilado, da un valor agregado a los productos vegetales, porque mejora su contenido nutricional, digestibilidad y palatabilidad; este último factor puede haber incrementado el consumo por parte de los animales, ocasionando el mayor consumo, mientras que el incremento en la digestibilidad tiene relación con la mayor eficiencia de uso de los nutrientes, representada en la mayor ganancia de peso del grupo alimentado con ensilaje de sauco.

Resultados opuestos fueron reportados por Partridge et al. (1985), quienes realizaron un estudio en conejos Nueza Zelanda blanco $\mathrm{x}$ California, con el fin de evaluar el efecto de sustituir cebada molida por el suministro a voluntad de ensilaje, conformado por una mezcla de Ryegrass perenne (Lolium perenne) 700; hierba timotea (Pbleum pratense) 210 y trébol blanco (Trifolium repens) $90 \mathrm{~g} / \mathrm{kg}$; al finalizar, encontraron que, por cada $1 \mathrm{~g}$ de cebada reemplazada por ensilaje, el consumo total de materia seca y la tasa de crecimiento disminuyeron, aproximadamente, $0,28 \mathrm{~g}$ y $0,20 \mathrm{~g} /$ día, respectivamente, frente al tratamiento control, lo que afectó directamente el factor de conversión alimenticia.

Lebas (1988) reporta que los conejos, generalmente, se alimentan ad libitum y pueden regular el consumo, siempre y cuando las concentraciones de energía de la dieta estén entre 9,2 y 13,0MJ de energía digestible (ED) por kilogramo. Para conejos en crecimiento $(0,5$ a 2,5kg), el consumo de energía debería ser de 900 a 1000kJ $\mathrm{ED} / \mathrm{kg} \mathrm{PV}^{0,75}$, es decir, aproximadamente, el doble del requerimiento de mantenimiento. Dicho punto sería interesante evaluarlo en futuras investigaciones, realizando ensayos de digestibilidad, tanto del sauco oreado como ensilado y así poder determinar el nivel de energía digestible consumida por los animales, cuando se sustituye, parcialmente, el concentrado del sistema productivo.

Por su parte, el sauco oreado presentó menores rendimientos productivos al comprarlo con el tratamiento control y el sauco ensilado; esto puede estar relacionado con la presentación del material, puesto que el sauco ensilado fue sometido previamente a un proceso de picado, lo que disminuyó significativamente su volumen, mientras que el sauco oreado no pasó por este proceso, haciendo su presentación más voluminosa. Frente a esto, Lebas (1988) menciona que el volumen de la dieta y la forma física en que se presenta el alimento influye sobre el valor de la energía dietética del resto de la dieta, debido a que, a menor volumen en la dieta, se incrementa la tasa de pasaje de la digesta, lo que puede reducir la digestibilidad de otros ingredientes que producen energía, sin afectar la digestibilidad aparente de la proteína. En este sentido, el tiempo de tránsito aumenta con el tamaño de las partículas, que contienen los constituyentes de la pared celular.

Por otra parte, tanto el rendimiento en canal como el índice viscerosomático (IV) no presentaron diferencias significativas entre tratamientos $(\mathrm{P}>0,05)$; sin embargo, el peso final de los conejos alimentados con sauco ensilado fue mayor, esto, a su vez, influyó en el peso de la canal, incidiendo positivamente sobre los parámetros económicos. 
El rendimiento en canal es una de las variables de mayor importancia económica cuando se comercializa el producto de esta manera, ya que se depende exclusivamente de la producción neta de carne y hueso, a diferencia de la venta en pie, donde los demás componentes (patas, cabeza, vísceras y piel) hacen parte del producto final y, por ende, se traducen en un menor costo del producto final. En el presente trabajo, el rango del rendimiento en canal observado (48,5 - 49,8\%) coincide con lo reportado por Peniche Ruz et al. (2010), al incluir Brosimum alicastrum (49,0 - 50,8\%) y por Leyva et al. (2012), con Artocarpus altilis (48,7 - 49,95\%), en el engorde de conejos. Esto indica, que la inclusión de suplementos no tradicionales no afecta el nivel de productividad de los individuos.

Parámetros económicos: En el costo total de alimentación de cada uno de los tratamientos, se evidencia que la dieta que fue suplementada con sauco ensilado presentó el mayor costo por concepto de alimentación; sin embargo, generó el mayor MBIP en los dos escenarios analizados (venta en pie y en canal), lo que se relaciona directamente con la mayor ganancia de peso obtenida, en este grupo de animales, lo cual, favorece económicamente al productor, al incrementar sus ingresos en un 9 y $13 \%$, cuando los animales son vendidos en pie y en canal, respectivamente, mientras que la dieta suplementada con sauco fresco presenta un menor MBIP que el tratamiento control, cuando los animales son vendidos en pie, cuya disminución, en el nivel de ingresos, sería del orden de $29 \%$, mientras que cuando se comercializan en canal, el ingreso incrementaría $1 \%$, con relación al tratamiento control, si se tuviera en cuenta únicamente los costos por concepto de alimentación (Tabla 2). Teniendo en cuenta estos resultados, se evidencia un mayor beneficio económico al comercializar los animales en canal, puesto que el precio de venta es mayor y se obtiene un mayor MBIP para el productor.

Tabla 2. Análisis de presupuestos parciales de conejos alimentados con sauco fresco y ensilado, durante 28 días.

\begin{tabular}{|c|c|c|c|}
\hline Concepto & Control & Oreado & Ensilado \\
\hline Costo concentrado ( $\$ / \mathrm{kg}$ de MS) & 1.667 & 1.667 & 1.667 \\
\hline Consumo concentrado (kg de MS) & 32,1 & 30,8 & 32,2 \\
\hline Costo sauco fresco $(\$ / \mathrm{kg}$ de MS) & & 364 & \\
\hline Consumo sauco fresco (kg de MS) & & 4,3 & \\
\hline Costo ensilaje ( $\$ / \mathrm{kg}$ de MS) & & & 401 \\
\hline Consumo ensilaje (kg de MS) & & & 5,4 \\
\hline Consumo total $(\mathrm{kg})$ & 32,1 & 35,1 & 37,6 \\
\hline Costo total de alimentación ( $\$ / \mathrm{kg}$ de MS/Jaula) & 53.429 & 52.956 & 55.851 \\
\hline Biomasa ganada (kg/tto) & 10,1 & 8,7 & 10,8 \\
\hline \multicolumn{4}{|c|}{ Análisis con venta de animales en pie } \\
\hline Costo/kg de carne producido $(\$ / \mathrm{kg})$ & 5.278 & 6.096 & 5.179 \\
\hline Precio de venta en pie $(\$ / \mathrm{kg})$ & 10.000 & 10.000 & 10.000 \\
\hline Ingreso total (\$) & 101.227 & 86.864 & 107.842 \\
\hline Margen bruto de ingreso parcial (\$) & 47.798 & 33.908 & 51.991 \\
\hline$\%$ Diferencial* & $0 \%$ & $-29 \%$ & $9 \%$ \\
\hline Valor diferencial $(\$)^{*}$ & - & $(13.890)$ & 4.192 \\
\hline \multicolumn{4}{|c|}{ Análisis con venta de animales en canal } \\
\hline Sumatoria Peso final/tto $(\mathrm{kg})$ & 21 & 21 & 23 \\
\hline Peso Canales/tto (kg) & 10 & 10 & 11 \\
\hline Precio de venta canal $(\$ / \mathrm{kg})$ & 16.000 & 16.000 & 16.000 \\
\hline Ingreso total $(\$)$ & 162.960 & 163.344 & 179.144 \\
\hline Margen bruto de ingreso parcial $(\$)$ & 109.531 & 110.388 & 123.293 \\
\hline$\%$ Diferencial* & $0 \%$ & $1 \%$ & $13 \%$ \\
\hline Valor diferencial $(\$)^{*}$ & - & 857 & 13.761 \\
\hline
\end{tabular}

* Con respecto a la dieta control. tto: tratamiento. 
La inclusión de harina del fruto y hojas del árbol del pan, como ingrediente y sustituto de alimentos comerciales, demuestra respuestas similares, confirmando que la venta en canal genera mayores utilidades, cuando se le compara con la venta en pie o peso vivo (Leyva et al. 2012). Esto, a la vez, permite el aprovechamiento de los subproductos generados en el proceso de posproducción, como la piel y las vísceras. De manera similar, la sustitución de ingredientes tradicionales, como la soya por harinas vegetales producidas en la región de producción, permite canales de mayor peso, comparado con animales alimentados convencionalmente (Demera, 2012).

El uso de fuentes distintas a los concentrados comerciales, como forrajes o ensilajes, se pueden convertir en un costo y una labor adicional dentro de los sistemas de producción, como se mostró en el presente trabajo; sin embargo, sus efectos positivos en el desempeño animal permiten obtener mayores ganancias económicas; de manera similar, otros autores reportan la disminución de los costos de producción y, por ende, la mejora de los indicadores de evaluación económica, como el valor actual neto (VAN) y la tasa interna de retorno (TIR), al incluir en las dieta para conejos Tithonia diversifolia y B. alicastrum (Quintero et al. 2007; Peniche Ruz et al. 2010). Estos resultados revisten de especial importancia para familias de recursos limitados, en aquellas áreas donde hay disponibilidad de fuentes vegetales alternativas, disminuyendo la dependencia por los alimentos comerciales.

Los ingredientes que constituyen los concentrados, en su mayoría, son importados, lo que explica su alto valor en el mercado, por lo que se hace importante no solo continuar investigando en suplementos alimenticios disponibles en el área de producción, sino también, en la sustitución de ingredientes, como la soya y el maíz que, aunque son de buena calidad nutricional, también aumentan el precio del concentrado. En este sentido, Demera (2012) expuso cómo el uso de harinas de maíz hidropónico y vaina de algarrobo permite la sustitución total de la soya, favoreciendo un mejor costo/beneficio, gracias al menor costo en la producción de alimento y la venta de canales de mayor peso.

Sumado a lo anterior, diferentes autores (Garcés et al. 2004; Demera, 2012), también indican que la inclusión de ensilados o el uso de harinas producidas "orgánicamente", favorecen la producción de un alimento natural, ecológico y más económico que los concentrados, lo que haría del silo y demás alternativas una opción viable, tal como se evidenció dentro del análisis de presupuestos parciales.

En conclusión, el ensilaje de sauco puede ser considerado como una alternativa viable para la suplementación alimenticia en los sistemas de producción cunícola, puesto que mejora algunos parámetros productivos, como la ganancia de peso y de conversión alimenticia, lo que repercute positivamente en el margen bruto de ingreso parcial, cuando se tienen en cuenta únicamente los costos asociados al alimento, con lo que el productor incrementa su nivel de ingresos, en torno al 9 y 13\%, cuando los animales son comercializados en pie y en canal, respectivamente; esto, al compararse con un sistema de producción basado solamente en concentrado, siendo más rentable vender los animales en canal, puesto que el precio de comercialización es mayor y se genera un mayor MBIP.
La literatura reporta la presencia de compuestos secundarios con posible efecto antiparasitario en forrajes y arbóreas (Waghorn, 2008); se sugiere realizar investigaciones para evaluar el posible efecto terapéutico del sauco en conejos. Igualmente, es de interés identificar la relación entre algunos índices fisiológicos, como el IV y las variables productivas como el consumo. Además, continuar con la evaluación de recursos alternativos, ya sean frescos o conservados, presentes en los sistemas de producción cunícula.

Agradecimientos. Al personal de la Unidad experimental El Remanso de la Universidad de Ciencias Aplicadas y Ambientales U.D.C.A y a los estudiantes del programa de Zootecnia y del programa de Medicina Veterinaria, de la asignatura investigación I, segundo semestre de 2017, quienes hicieron posible la realización de este trabajo, con su apoyo en el cuidado de los animales y recolección de datos. Conflicto de interés: El manuscrito fue preparado y revisado con la participación de todos los autores, quienes declaramos que no existe ningún conflicto de intereses que ponga en riesgo la validez de los resultados presentados. Financiación: Este estudio fue financiado por la Universidad de Ciencias Aplicadas y Ambientales U.D.C.A.

\section{REFERENCIAS}

1. BARRIAL, A.M.; BARRIAL, A.M. 2011. La educación alimentaria y nutricional desde una dimensión sociocultural como contribución a la seguridad alimentaria y nutricional. Contribuciones a las Ciencias Sociales. Disponible desde internet en: http://www.eumed.net/rev/cccss/16/bmbm. html (con acceso 03/11/2018).

2. CÁRDENAS, C.A.; ROCHA, C.; MORA, J. 2011. Productividad y preferencia de forraje de vacas lecheras pastoreando un sistema silvopastoril intensivo de la zona alto Andina de Roncesvalles, Tolima. Rev. Colombiana de Ciencia Animal. $4(1): 29-35$.

3. CARVAJAL, T.; CUESTA, A. 2016. Conservación y composición nutricional del follaje de saúco (Sambucus nigra). Pastos y Forrajes. 39(2):125-132.

4. CARVAJAL, T.; LAMELA, L.; CUESTA, A. 2012. Evaluación de las arbóreas Sambucus nigra y Acacia decurrens como suplemento para vacas lecheras en la Sabana de Bogotá, Colombia. Pastos y Forrajes. 35(4):417-429.

5. CULLERE, M.; DALLE ZOTTE, A. 2018. Rabbit meat production and consumption: State of knowledge and future perspectives. Meat Science. 143:137-146. https:// doi.org/10.1016/j.meatsci.2018.04.029

6. DALLE ZOTTE, A.; SZENDRÖ, Z. 2011. The role of rabbit meat as functional food. Meat Science. 88:319-331. https:// doi.org/10.1016/j.meatsci.2011.02.017 
7. DEMERA, J. 2012. Utilización de harinas de maíz hidropónico deshidratada y vaina de algarrobo en reemplazo parcial y total de la soya en la alimentación de conejos neozelandés en la etapa de crecimiento y engorde. Revista la Técnica. 8:48-57.

8. GARCÉS, A.M.; BERRIO, L.; RUÍZ, S.; SERNA, J.G.; BUILES, A.F. 2004. Ensilaje como fuente de alimentación para el ganado. Rev. Lasallista de Investigación. 1(1):66-71.

9. GARCÍA, D.; MEDINA, M.; DOMÍNGUEZ, C.; BALDIZÁN, A.; HUMBRÍA, J.; COVA, L. 2006. Evaluación química de especies no leguminosas con potencial forrajero en el estado Trujillo, Venezuela. Zootecnia Tropical. 24(4):401415.

10. GIDENNE, T.; JEHL, L. 1996. Replacement of starch by digestible fiber in the feed for the growing rabbit. 1. Consequences for digestibility and rate of passage. Animal Feed Science Technology. 61(1-4):183-192. https://doi. org/10.1016/0377-8401(95)00937-X

11. LEBAS, F. 1988. Rabbits. Livestock Production Science. 19:289298.

12. LEBAS, F.; COUDERT, P.; DE ROCHAMBEAU, H.; THÉBAULT, R.G. 1996. El Conejo Cria y patología (nueva versión revisada). Colección FAO: Producción y sanidad animal, 19. Roma Italia. 269p.

13. LEYVA, C.; VALDIVIÉ, M.Y.; ORTIZ, A. 2012. Utilización de harina de frutos y hojas del árbol del pan (Artocarpus altilis) en la ceba de conejos Nueva Zelanda Blanco. Pastos y Forrajes. 35(4):443-452.

14. MARTÍNEZ, B.R.; MARTÍNEZ, R.N.; MARTÍNEZ, M.M.V. 2011. Diseño de Experimentos en Ciencias Agropecuarias y Biológicas con SAS, SPSS, R y STATISTIX. Tomo I. Primera edición, Bogotá, D. C: Fondo Nacional Universitario, I.A.C. 399p.

15. MBANYA，J.N.; NDOPING，B.N.; MAFENI，J.M.; FOMUNYAM, D.W. 2005. The effect of different protein sources and their combination on the performance of growing rabbits in tropical conditions. Livestock Research for Rural Development 17(3):32.

16. MINISTERIO DE AMBIENTE, VIVIENDA Y DESARROLLO TERRITORIAL, MAVDT. 2009. Caracterización del mercado colombiano de plantas medicinales y aromáticas. 32p.

17. MORA-VALVERDE, D. 2010. Usos de la morera (Morus alba) en la alimentación del conejo. El rol de la fibra y la proteína en el tracto digestivo. Agronomía Mesoamericana. 21(2):357366. https:/doi.org/10.15517/am.v21i2.4900
18. NOVA, L.B.; CHAMORRO, D.R.; CARULLA, J.E. 2005. Caracterización química y nutricional de seis arbóreas del trópico de altura para la suplementación de rumiantes. Informe técnico proyecto "Desarrollo de alternativas silvopastoriles para sistemas de producción de bovinos de leche en los Altiplanos de Cundinamarca". SENA, UDCA, USALLE, CORPOICA, UNAD, AGAP. Colombia. 12p.

19. OLIVARES, R.; GÓMEZ, M.A.; SCHWENTESIUS, R.; CARRERA B. 2009. Alternativas a la producción y mercadeo para la carne de conejo en Tlaxcala, México. Región y Sociedad. 21(46):191-207. https://doi.org/10.22198/ rys.2009.46.a482

20. PARTRIDGE, G.G.; ALLAN, S.J.; FINDLAY, M. 1985. Studies on the nutritive value of roots, cabbage and grass silage for growing commercial rabbits. Animal Feed Sci. Technol. 13:299-311. https://doi.org/10.1016/03778401(85)90032-X

21. PENICHE RUZ, J.A.; REJÓN ÁVILA, M.J.; VALENCIA HEREDIA, E.R.; PECH MARTÍNEZ, V.C. 2010. Análisis de rentabilidad de dos alternativas de alimentación no convencionales en la producción de conejos en el municipio de Tixpehual, Yucatán, México. Rev. Mexicana de Agronegocios. 27:411-418.

22. QUINTERO, V.; GARCÍA, G.; PELÁEZ, A. 2007. Evaluación de harina de botón de oro en dietas para conejos en etapa de crecimiento. Acta Agronómica. 56(4):203-206.

23. SÁNCHEZ, L.; AMADO, G.M.; CRIOLLO, P.J.; CARVAJAL, T.; ROA, J.; CUESTA, A.; CONDE, A.; UMAÑA, A.; BERNAL, L.M.; BARRETO L. 2009. El sauco (Sambucus nigra) como alternativa silvopastoril en el manejo sostenible de praderas en el trópico alto colombiano. Corpoica. Ed. Produmedios. Colombia. 55p.

24. SAS, Institute. 2000. SAS/STAT User's Guide: Statistics. Release 6.04 Edition. SAS Institute Inc.

25. SEIJA, C.E. 2011. Revisión de experiencias urbanas y periurbanas de cría animal como alternativa de seguridad alimentaria. Rev. de Investigación Agraria y Ambiental. 2(2):51-63.

26. WAGHORN, G. 2008. Beneficial and detrimental effects of dietary condensed tannins for sustainable sheep and goat production - Progress and challenges. Animal Feed Science and Technology. 147(1-3):116-139. https://doi. org/10.1016/j.anifeedsci.2007.09.013 